九州大学学術情報リポジトリ

Kyushu University Institutional Repository

\title{
RATES OF CONVERGENCE IN CENTRAL LIMIT THEOREM FOR MARTINGALE DIFFERENCES
}

Kato, Yutaka

Department of Industrial Engineering, College of Engineering, Hosei University

https://doi.org/10.5109/13119

出版情報 : 統計数理研究. 18 (1/2)，pp. 1-8，1978-03. Research Association of Statistical Sciences

バージョン :

権利関係 : 


\title{
RATES OF CONVERGENCE IN CENTRAL LIMIT THEOREM FOR MARTINGALE DIFFERENCES
}

\author{
By \\ Yutaka KATO* \\ (Received October 30, 1976; revised August 1, 1977)
}

In this note we shall give an estimate to the rate of convergence in central limit theorem for bounded martingale difference sequence, which is a better estimate than Ibragimov's one [3]. Our method is based on Skorohod representation theorem (straightforword probabilistic methods).

Let $\left\{X_{i} ; i=1,2, \cdots\right\}$ be a sequence of martingale differences with $P\left\{\left|X_{i}\right| \leqq C\right\}=1$ for all $i$. We define the random variables

$$
\begin{aligned}
& s_{0}^{2}=E\left\{X_{1}^{2}\right\} \\
& s_{j}^{2}=E\left\{X_{j+1}^{2} \| X_{j}, \cdots, X_{1}\right\}, \quad j=1,2, \cdots
\end{aligned}
$$

and we also define the random indexes $\nu_{n}$ by the inequalities

$$
s_{0}^{2}+\cdots+s_{\nu^{-1}}^{2}<n \leqq s_{0}^{2}+\cdots+s_{\nu_{n}}^{2}, \quad n=1,2, \cdots
$$

Finally, we define

$$
S_{n}=X_{1}+\cdots+X_{\nu_{n}} .
$$

I. A. Ibragimov [3] showed that if $\sum_{j=0}^{\infty} s_{j}^{2}$ diverges with probability 1 , then

$$
\begin{aligned}
& \sup _{x}\left|P\left\{\frac{S_{n}}{\sqrt{n}} \leqq x\right\}-\Phi(x)\right| \\
\leqq & 2 \frac{C^{1 / 2}}{\sqrt[4]{n}}\left(1+\frac{3}{2} \frac{C}{\sqrt{n}}+\frac{1}{3} \frac{C^{2}}{n}\right),
\end{aligned}
$$

where $\Phi(x)$ is a standard normal distribution function. We give the theorem which deals with the suggestions given to me by Ibragimov [4] in private conversations.

THEOREM: Let $\left\{X_{i} ; i=1,2, \cdots\right\}$ be a sequence of martingale differences with $P\left\{\left|X_{i}\right| \leqq C\right\}=1$. If

$$
\sum_{j=0}^{n} s_{j}^{2}(\omega) \asymp f(n) \quad \text { uniformly }
$$

* Department of Industrial Engineering, College of Engineering, Hosei University, Tokyo. 
for some monotone increasing function $f(n)$ such that

$$
\frac{f^{-1}(n)}{n}=o(\log n), \quad \frac{f(n)}{n}=0(1),
$$

then there exists a constant $M$ such that for sufficiently large $n$

$$
\sup _{x}\left|P\left\{\frac{S_{n}}{\sqrt{n}} \leqq x\right\}-\Phi(x)\right| \leqq M \frac{(\log n)^{3}}{\sqrt{n}} .
$$

The constant $M$ depends only on the constants $C, \beta$ and $\gamma$,

where $\quad \beta=\liminf _{n \rightarrow \infty} \underset{\omega}{\operatorname{ess} . i n f} \frac{1}{f(n)} \sum_{j=0}^{n} s_{j}^{2}(\omega), \quad \gamma=\limsup _{n \rightarrow \infty} \underset{\omega}{\text { ess. } \sup } \frac{1}{f(n)} \sum_{j=0}^{n} s_{j}^{2}(\omega)$.

REMARK: The condition (2) of Theorem means that

$$
\begin{aligned}
& 0<\liminf _{n \rightarrow \infty} \underset{\omega}{\operatorname{linf}} \frac{1}{f(n)} \sum_{j=0}^{n} s_{j}^{2}(\omega) \\
& \leqq \limsup _{n \rightarrow \infty} \text { ess. } \sup \frac{1}{f(n)} \sum_{j=0}^{n} s_{j}^{2}(\omega)<\infty .
\end{aligned}
$$

In proving the theorem, we shall use the following three lemmas. The first one is a Skorohod representation theorem for martingale differences which was given by Strassen [1].

\section{LEMma 1: Let}

$$
X_{1}, X_{2}, \cdots
$$

be random variables such that for all $n, E\left\{X_{n}^{2} \| X_{n-1}, \cdots, X_{1}\right\}$ exists and $E\left\{X_{n} \| X_{n-1}, \cdots\right.$, $\left.X_{1}\right\}=0$ a.e. Then, without loss of generality, there is a Brownian motion $w(t)$ together with a sequence of non-negative random variables $T_{1}, T_{2}, \cdots$ such that

$$
\sum_{i=1}^{n} X_{i}=w\left(\sum_{i=1}^{n} T_{i}\right) \text { a.e., }
$$

for all $n$. Moreover, if $\mathscr{B}_{n}$ is generated by $X_{1}, \cdots, X_{n}$ and $w(t)$ for $0 \leqq t \leqq \sum_{i=1}^{n} T_{i}$, then the following hold.

(i) $T_{n}$ is $\mathscr{B}_{n}$-measurable.

(ii) For any $s>0, w\left(\sum_{i=1}^{n} T_{i}+s\right)-w\left(\sum_{i=1}^{n} T_{i}\right)$ is independent of $\mathscr{B}_{n}$.

(iii) $E\left\{T_{n} \| \mathscr{B}_{n-1}\right\}$ exists and $E\left\{T_{n} \| \mathscr{B}_{n-1}\right\}=E\left\{X_{n}^{2} \| \mathscr{B}_{n-1}\right\}=E\left\{X_{n}^{2} \| X_{n-1}, \cdots, X_{1}\right\}$ a.e.

(iv) If $k$ is a real number $>1$ and $E\left\{X_{n}^{2 k} \| X_{n-1}, \cdots, X_{1}\right\}$ exists, then $E\left\{T_{n}^{k} \| \mathscr{B}_{n-1}\right\}$ exists too, and further $E\left\{T_{n}^{k} \| \mathscr{B}_{n-1}\right\} \leqq L_{k} E\left\{X_{n}^{2 k} \| \mathscr{B}_{n-1}\right\} \leqq L_{k} E\left\{X_{n}^{2 k} \| X_{n-1}, \cdots, X_{1}\right\}$ a. e., where each $L_{k}$ is a constant which depends only on $k$. 
The phrase 'without loss of generality' in the above lemma is used in a specific sense, namely: there is a new probability space $(\hat{\Omega}, \hat{\mathcal{B}}, \hat{P})$ on which random variables

$$
\hat{X}_{1}, \hat{X}_{2}, \cdots
$$

are defined in such a way that the both sequences (4) and (5) have the same distribution, and the assertions in the above lemma (where now 'without loss of generality' is to be omitted) remain valid for the $\hat{X}_{n}\left(w(t)\right.$ and the $T_{n}$ are of course defined on the new space). In this note, we shall assume that the new probability space $(\hat{\Omega}, \hat{\mathcal{B}}, \hat{P})$ satisfies the following conditions: (I) There exists a sequence of independent Brownian motions $\left\{B_{n}(t), n \geqq 1\right\}$ on $(\hat{\Omega}, \hat{\mathcal{B}}, \hat{P})$ such that $B_{n}(t)$ converges with probability 1 to Brownian motion $B(t)$. (II) There exist random variables independent of all the $B_{n}(t)$, $Y_{1}, Y_{2}, \cdots$ on $(\hat{\Omega}, \hat{\mathcal{B}}, \hat{P})$ which are pairwise independent and distributed uniformly over the interval $[0,1]$.

Hereafter, we shall discuss on the new probability space, and we shall write $X_{1}, X_{2}, \cdots$ in place of $(5)$ and $(\Omega, \mathscr{B}, P)$ in place of $(\hat{\Omega}, \hat{\mathcal{B}}, \hat{P})$.

The next two lemmas are the generalizations of results of Skorohod [2] on sums of independent random variables to martingales. The proofs can be obtained in a way similar to Skorohod's one and are omitted.

LEMma 2: For each $n$, suppose that $\mathcal{B}_{n, 1}, \mathcal{B}_{n, 2}, \cdots$ are non-decreasing sequence of $\sigma$-algebras and that $\eta_{n, 1}, \eta_{n, 2}, \cdots$ are random variables such that for each $i, \eta_{n, i}$ is $\mathscr{B}_{n, i}$-measurable, $E\left\{\eta_{n, i} \| \mathscr{B}_{n, i-1}\right\}=0$ a.e., $E\left\{\eta^{2}{ }_{n, i} \| \mathscr{B}_{n, i-1}\right\} \leqq H_{1} / n$ and $E\left\{\eta^{4}{ }_{n, i}\right\} \leqq H_{2} / n^{2}$, where $\mathscr{B}_{n, 0}$ is a trivial $\sigma$-algebra. If $\nu_{n}(\omega)$ is a non-negative integer-valued random variable such that ess. sup $\nu_{n}(\omega) \leqq g(n)$ for some positive integer-valued function $g(n)$, then

$$
\begin{aligned}
& P\left\{\left|\sum_{i=1}^{\nu n} \eta_{n, i}\right|>2 \log n\right\} \\
& \quad \leqq \frac{g(n)}{n^{2}}\left(2 \exp \left\{\left(1+\frac{e}{2}\right) H_{1} \frac{g(n)}{n}\right\}+H_{2}\right) .
\end{aligned}
$$

LEMma 3: For each n, suppose that $\mathscr{B}_{n, 1}, \mathscr{B}_{n, 2}, \cdots$ are non-decreasing sequence of $\sigma$-algebras and that $\eta_{n, 1}, \eta_{n, 2}, \cdots$ are random variables such that for each $i, \eta_{n, i}$ is $\mathcal{B}_{n, i}$-measurable, $E\left\{\eta_{n, i} \| \mathcal{B}_{n, i-1}\right\}=0$ a. e. and $E\left\{\eta_{n, i}^{k} \| \mathcal{B}_{n, i-1}\right\} \leqq H_{k} /(\log n)^{k}, \quad k=2,3, \cdots$, where $H_{k} \leqq A^{k}$ for some constant $A$ and $\mathscr{B}_{n}$ is a trivial $\sigma$-algebra. Then we have

$$
\begin{aligned}
& P\left\{\left|\sum_{i=1}^{\left[(\log n)^{8]}\right.} \eta_{n, i}\right|>\left(\left(1+\frac{e}{2}\right) H_{2}+3\right) \log n\right\} \\
& \leqq \frac{2}{n^{3}}+\frac{A^{3 n}}{(\log n)^{3 n-3}} .
\end{aligned}
$$

We can now prove our theorem as follows. It follows from Lemma 1 that there is a Brownian motion $w(t)$ together with a sequence of non-negative random variables $T_{1}, T_{2}, \cdots$ such that for each $k$ 


$$
\sum_{i=1}^{k} \xi_{n, i}=w\left(\sum_{i=1}^{k} T_{i}\right) \text { a.e., }
$$

where $\xi_{n, i}=\frac{1}{\sqrt{n}} X_{i} . \quad$ Moreover, if $\mathscr{B}_{n, i}=\mathscr{B}\left\{\xi_{n, 1}, \cdots, \xi_{n, i}, w(t), 0 \leqq t \leqq \sum_{j=1}^{i} T_{j}\right\}, \quad$ then we have

$$
E\left\{T_{i}^{m} \| \mathcal{B}_{n, i-1}\right\} \leqq L_{m}\left(\frac{C}{\sqrt{n}}\right)^{2 m} \text { a.e., }
$$

since, by hypothesis, $P\left\{\left|\xi_{n, i}\right| \leqq \frac{C}{\sqrt{n}}\right\}=1$.

We denote by $Q_{n}(x)$ the probability

$$
\begin{aligned}
Q_{n}(x) & =P\left\{\frac{S_{n}}{\sqrt{n}} \leqq x\right\} \\
& =P\left\{\xi_{n, 1}+\cdots+\xi_{n, \nu_{n}} \leqq x\right\} \\
& =P\left\{w\left(\sum_{i=1}^{\nu_{n}} T_{i}\right) \leqq x\right\}
\end{aligned}
$$

and by $Q(x)$ the probability

$$
Q(x)=P\{w(1) \leqq x\}
$$

We define $\eta_{n, i}=n^{1 / 2}\left(T_{i}-E\left\{T_{i} \| \mathscr{B}_{n, i-1}\right\}\right)$, and $\zeta_{n, i}=\sum_{j=1}^{i} \eta_{n, j} . \quad$ Then we have

$$
w\left(T_{1}+\cdots+T_{\nu_{n}}\right)=w\left(\frac{\zeta_{n, \nu_{n}}}{\sqrt{n}}+\sum_{i=1}^{\nu_{n}} E\left\{T_{i} \| \mathscr{B}_{n, i-1}\right\}\right) .
$$

Thus

$$
Q_{n}(x)=P\left\{w\left(\frac{\zeta_{n, \nu_{n}}}{\sqrt{n}}+\sum_{i=1}^{\nu_{n}} E\left\{T_{i} \| \mathscr{B}_{n, i-1}\right\}\right) \leqq x\right\}
$$

From the property of the stopping time $T_{k+1}$, it follows that for any $s \in\left[\sum_{i=1}^{k} T_{i}, \sum_{i=1}^{k+1} T_{i}\right]$

$$
\left|w(s)-w\left(\sum_{i=1}^{k} T_{i}\right)\right| \leqq C / n^{1 / 2}
$$

Therefore, we have

(8) $P\left\{w(s) \leqq x-\frac{(\log n)^{3}}{\sqrt{n}} C\right.$, for some $\left.s \in\left[\sum_{i=1}^{\nu_{n}} T_{i},{ }^{\left.\nu_{n}+[\log n)^{33}\right]} T_{i}\right]\right\} \leqq Q_{n}(x)$

$$
\leqq P\left\{w(s) \leqq x+\frac{(\log n)^{3}}{\sqrt{n}} C, \quad \text { for any } \quad s \in\left[\sum_{i=1}^{\nu_{n}} T_{i},{ }_{i=1}^{\nu_{n}+\left[(\log n)^{3}\right]} T_{i}\right]\right\} .
$$


From the conditions (2) and (3) of Theorm, it follows that for sufficiently large $n$

$$
\sum_{i=\nu_{n}+1}^{\left.\nu_{n}+[\log n)^{3}\right]} E\left\{T_{i} \| \mathscr{B}_{n, i-1}\right\} \geqq \frac{\beta}{6} \frac{(\log n)^{3}}{n \log \log n},
$$

where $\beta=\liminf _{n \rightarrow \infty} \underset{\omega}{\text { ess.inf }} \frac{1}{f(n)} \sum_{j=0}^{n} s_{j}^{2}(\omega)$. We define $\quad \bar{\eta}_{n, i}=\frac{n^{1 / 2}}{\log n} \eta_{n, i}$, then the variables $\bar{\eta}_{n, i}$ satisfy the conditions of Lemma 3. Furthermore, we observe that event $\left\{\nu_{n}=k\right\}$ is $\mathscr{B}_{n, k}$-measurable, and hence

$$
E\left\{\chi_{k} \bar{\eta}_{n, j} \| \mathscr{B}_{n, j-1}\right\}=\chi_{k} E\left\{\bar{\eta}_{n, j} \| \mathscr{B}_{n, j-1}\right\}=0 \text { a. e. }
$$

for all $k \leqq j-1$, where $\chi_{k}$ is a indicator function of $\left\{\nu_{n}=k\right\}$. Thus there exists a set $B$ such that, for sufficiently large $n, P\left(B^{c}\right) \leqq 3 / n$ and

$$
\sup _{\omega \in B}\left|\frac{1}{\sqrt{n}}{ }_{i=\nu_{n}+1}^{\nu_{n}+[(\log n) 3]} \eta_{n, i}\right| \leqq \frac{(\log n)^{2}}{n}\left(\left(1+\frac{e}{2}\right) C^{4}+3\right) .
$$

Therefore, we have

$$
\delta_{n}=\inf _{\omega \in B} \sum_{i=\nu_{n}+1}^{\nu_{n}+[(\log n) 3]} T_{i}>\frac{(\log n)^{2}}{n},
$$

so that, it follows from (8) that

$$
\begin{aligned}
Q_{n}(x) & \leqq \frac{3}{n} \\
& +P\left\{w(s) \leqq x+\frac{(\log n)^{3}}{\sqrt{n}} C, \quad \text { for any } \quad s \in\left[\sum_{i=1}^{\nu_{n}} T_{i}, \sum_{i=1}^{\nu_{n}} T_{i}+\delta_{n}\right]\right\} .
\end{aligned}
$$

Furthermore, from the property (I) and (II) of the new probability space, there exists a Brownian motion $w_{1}(t)$ independent of $w(t)$ such that

$$
P\left\{\left|\sum_{i=1}^{\nu_{n}} T_{i}-\sum_{i=1}^{\nu_{n}} T_{i}^{(1)}\right|>\frac{1}{n}\right\}<\frac{1}{n},
$$

where random variables $T_{1}^{(1)}, T_{2}^{(1)}, \cdots$ are the stopping times for $w_{1}(t)$ determined by Skorohod representation theorem (Lemma 1), see Basu [5]. Consequently, we have for sufficiently large $n$

$$
Q_{n}(x) \leqq P\left\{w(\tau) \leqq x+\frac{(\log n)^{3}}{\sqrt{n}} C\right\}+\frac{4}{n}
$$

where the random variable $\tau=\left(\sum_{i=1}^{\nu n} T_{i}^{(1)}+\frac{\delta_{n}}{2}\right)$ is independent of Brownian motion $w(t)$ and

$$
\tau \in\left[\sum_{i=1}^{\nu_{n}} T_{i}, \sum_{i=1}^{\nu_{n}} T_{i}+\delta_{n}\right]
$$

Since $w(\tau)$ and $\sqrt{\tau} w(1)$ have the same distribution for fixed $\tau$, it follows that 


$$
Q_{n}(x) \leqq P\left\{\sqrt{\tau} w(1) \leqq x+\frac{(\log n)^{3}}{\sqrt{n}} C\right\}+\frac{4}{n} .
$$

From the condition (2) of Theorem, it follows that for sufficiently large $n$

$$
\text { ess. } \sup \nu_{n}(\omega) \leqq\left[f^{-1}\left(\frac{2}{\beta} n\right)\right]+1 \leqq 2 f^{-1}\left(\frac{2}{\beta} n\right) \text {. }
$$

Furthermore, the variables $\eta_{n, i}$ satisfy the conditions of Lemma 2 , then we have

$$
\begin{aligned}
& P\left\{\left|\zeta_{n, \nu_{n}}\right|>2 \log n\right\} \\
& \quad \leqq \frac{2}{n^{2}} f^{-1}\left(\frac{2}{\beta} n\right) \cdot\left(\exp \left\{\frac{C_{1}}{n} f^{-1}\left(\frac{2}{\beta} n\right)\right\}+C_{2}\right),
\end{aligned}
$$

where $C_{1}=2\left(1+\frac{e}{2}\right) C^{4}$ and $C_{2}=C^{8}$. From the definition of $\nu_{n}$ and Lemma 1 , we have

$$
1-\frac{C^{2}}{n} \leqq \sum_{i=1}^{\nu_{n}} E\left\{T_{i} \| \mathscr{B}_{n, i-1}\right\}<1
$$

Therefore, we have the following inequality on $B \cap\left\{\left|\zeta_{n, \nu_{n}}\right| \leqq 2 \log n\right\}$

$$
1-M_{1} \frac{\log n}{\sqrt{n}} \leqq \tau \leqq 1+M_{1} \frac{\log n}{\sqrt{n}}
$$

where $M_{1}$ is a constant depending only on $C$ and $\gamma, \quad \gamma=\underset{n \rightarrow \infty}{\limsup } \operatorname{ess.\operatorname {sup}} \frac{1}{f(n)} \sum_{j=0}^{n} s_{j}^{2}(\omega)$. Then, from (10) and (11), we have for sufficiently large $n$

$$
\begin{aligned}
Q_{n}(x) & \leqq P\left\{w(1) \leqq x+2 M_{1}|x| \frac{\log n}{\sqrt{n}}+M_{2} \frac{(\log n)^{3}}{\sqrt{n}}\right\} \\
& +\frac{2}{n^{2}} f^{-1}\left(\frac{2}{\beta} n\right) \cdot\left(\exp \left\{\frac{C_{1}}{n} f^{-1}\left(\frac{2}{\beta} n\right)\right\}+C_{2}\right)+\frac{8}{n},
\end{aligned}
$$

where $M_{2}$ is a constant depending only on $C$ and $\gamma$.

In an analogous fashion, we can show that

$$
\begin{aligned}
Q_{n}(x) & \geqq P\left\{w(1) \leqq x-2 M_{1}|x| \frac{\log n}{\sqrt{n}}-M_{2} \frac{(\log n)^{3}}{\sqrt{n}}\right\} \\
& -\frac{2}{n^{2}} f^{-1}\left(\frac{2}{\beta} n\right) \cdot\left(\exp \left\{\frac{C_{1}}{n} f^{-1}\left(\frac{2}{\beta} n\right)\right\}+C_{2}\right)-\frac{8}{n} .
\end{aligned}
$$

Therefore, we have for sufficiently large $n$

$$
\begin{aligned}
& \left|Q_{n}(x)-Q(x)\right| \\
& \quad \leqq P\left\{x-2 M_{1}|x| \frac{\log n}{\sqrt{n}}-M_{2} \frac{(\log n)^{3}}{\sqrt{n}}<w(1) \leqq x\right\}
\end{aligned}
$$




$$
\begin{aligned}
& +P\left\{x<w(1) \leqq x+2 M_{1}|x| \frac{\log n}{\sqrt{n}}+M_{2} \frac{(\log n)^{3}}{\sqrt{n}}\right\} \\
& +-\frac{4}{n^{2}} f^{-1}\left(\frac{2}{\beta} n\right) \cdot\left(\exp \left\{\frac{C_{1}}{n} f^{-1}\left(\frac{2}{\beta} n\right)\right\}+C_{2}\right)+\frac{16}{n}
\end{aligned}
$$

But, it follows that for sufficiently large $n$

$$
\begin{aligned}
& P\left\{x-2 M_{1}|x| \frac{\log n}{\sqrt{n}}-M_{2} \frac{(\log n)^{3}}{\sqrt{n}}<w(1) \leqq x\right\} \leqq M_{3} \frac{(\log n)^{3}}{\sqrt{n}} \\
& P\left\{x<w(1) \leqq x+2 M_{1}|x| \frac{\log n}{\sqrt{n}}+M_{2} \frac{(\log n)^{3}}{\sqrt{n}}\right\} \leqq M_{3} \frac{(\log n)^{3}}{\sqrt{n}},
\end{aligned}
$$

where $M_{3}$ is a constant depending only on $C$ and $\gamma$. Consequently, it follows from (6), (7), (12) and (13) that

$$
\begin{aligned}
\sup _{x} \mid & P\left\{\frac{S_{n}}{\sqrt{n}} \leqq x\right\}-\Phi(x) \mid \\
\leqq & 2 M_{3} \frac{(\log n)^{3}}{\sqrt{n}}+\frac{16}{n} \\
& +\frac{4}{n^{2}} f^{-1}\left(\frac{2}{\beta} n\right) \cdot\left(\exp \left\{\frac{C_{1}}{n} f^{-1}\left(\frac{2}{\beta} n\right)\right\}+C_{2}\right) .
\end{aligned}
$$

Therefore, it follows from the condition (3) of Theorem that there exists a constant $M$ (depending only on $C, \beta$ and $\gamma$ ) such that

$$
\sup _{x}\left|P\left\{\frac{S_{n}}{\sqrt{n}} \leqq x\right\}-\Phi(x)\right| \leqq M \frac{(\log n)^{3}}{\sqrt{n}} .
$$

The proof of Theorem is now complete.

REMARKS: (1) We can prove in the same manner that

$$
\sup _{x}\left|P\left\{\frac{S_{n}}{\sqrt{n}} \leqq x\right\}-\Phi(x)\right|=0\left(\frac{(\log n)^{2+\delta}}{\sqrt{n}}\right)
$$

for any $\delta>0$.

(2) In the same manner, we can give an estimate to the rate of convergence in central limit theorem for a class of dependent $R^{k}$-valued random variables, see Y. Kato [6].

Acknowledgements: The author would like to express his thanks to Prof. M. Kowada of University of Tsukuba for his encouragement and suggestions. 


\section{References}

[1] V. STRASSEN, Almost sure behaviour of sums of independent random variables and martingales, Proceedings of the Fifth Berkeley Symposium on Mathematical Statistics and Probability, University of Carifornia Press, Vol. II, Part I (1966), 315-343.

[2] A.V. Skorohod, Studies in the Theory of Random Processes, Addison-Wesley, Massachusetts, 1965.

[3] I.A. Ibragimov, A central limit theorem for a class of dependent random variables, Theory of Probability and it's Applications (1963), 8, 83-89.

[4] I. A. Ibragimov, Private conversation, (1975).

[5] A.K. BAsu, A note on Strassen's version of the law of the iterated logarithm, Proceedings of American Mathematical Society, 41 (1973), 596-601.

[6] Y. Kato, Rates of convergence in central limit theorem for a class of dependent $R^{k}$-valued random variables, Bulletin of the College of Engineering, Hosei University, 13 (1977), 19-24. 